\title{
Study in Pursuing Differentiated Market
}

\author{
Yanwei Shi ${ }^{1}$, Chunhui Ma ${ }^{2}$ \\ ${ }^{1}$ School of Intelligent Science and Information Engineering in Xi'an Peihua University, Xi'an, China 710125 \\ ${ }^{2}$ School of Science in Xi'an University of Architecture and Technology, Xi'an, China 710055
}

Keywords: pursuing differentiated market; duopoly model; equilibrium

\begin{abstract}
The main purpose of this paper is to study the equilibriums in pursuing differentiated market. At first, the concept of pursuing differentiated market is proposed, and the equilibriums are discussed in same type of products with or without fixed costs. Then the equilibriums are analyzed in different types of products with same or different alternative coefficients. Finally, characters of the equilibrium of pure strategy are shown in general form matrix game.
\end{abstract}

\section{Introduction}

Duopoly model is a common market model in economic theory. In free market, if the market demand of a certain product is controlled by two enterprises, this phenomenon is called duopoly. The model used to describe the phenomenon of duopoly is called duopoly model [1-5]. In 1838, French economist Cournot first studied this market structure and established the Cournot duopoly model. Later, von Neumann and Oscar Morgenstern discussed the equilibrium in this model. The discussion of Nash equilibrium has been one of the topics in economics and game theory [6-8].

With the development of economy and enrichment of social material, people's consumption behavior in the market is also changing to a certain extent. Every consumer hopes to fully reflect their sense of self-existence and publicize their own personality through their own consumption behavior, making the individualized differences more obvious. So, the pursuing differentiated market is very necessary.

The pursuing differentiated market means that every consumer in the market, in pursuit of differentiation, always prefers low yield products in the process of freely choosing the same kind of products. This market environment is not uncommon in everyday life. For example, under the condition of affordable consumption, young people prefer to low yield iphones when choosing mobile phones. Another example, when buying luxury goods, consumers like to choose the low yield brands or limited editions of the same brand.

In this paper, the equilibriums are studied in pursuing differentiated market. For same products produced by duopoly, it is discussed that same type of products with or without fixed costs and different types of products with same or different alternative coefficients. Lastly, characters of the equilibrium of pure strategy are shown in general form matrix game.

\section{The equilibriums in pursuing differentiated market}

In the pursuing differentiated market, we suppose that there are only two enterprises, enterprise 1 and enterprise 2, for the production and sale of a certain product. Similar to Cournot model. Each enterprise can only choose their own yield independently, $q_{1}$ and $q_{2}$, respectively. Let $\left(q_{1}^{*}, q_{2}^{*}\right)$ is the equilibrium in this model.

\subsection{Same type of products}

Since the market is pursuing differentiated market, the consumer prefers to choose the product with low yield. So, the market price of the product is $p\left(q_{1}, q_{2}\right)=a-\min \left(q_{1}, q_{2}\right)$. 
A1) Without fixed costs. If the cost of production of both enterprises is $c$, and the fixed cost, such as plants, equipments, etc, the profit function of enterprise $i$ is

$$
u_{i}\left(q_{i}, q_{j}\right)= \begin{cases}\left(a-q_{i}\right) q_{i}-c q_{i} & q_{i}<q_{j} \\ \frac{1}{2}\left[\left(a-q_{i}\right) q_{i}-c q_{i}\right] & q_{i}=q_{j} \\ 0 & q_{i}>q_{j}\end{cases}
$$

where $\{i, j\}=\{1,2\}$. Because enterprises always seek profits and maximize profits, by the profit function, it can be seen that $q_{1}^{*}=q_{2}^{*}$. Otherwise, enterprise with higher yield will have the desire to change yield, and it is not equilibrium. Thus, the profit of enterprise $i$ is $u_{i}\left(q_{i}, q_{j}\right)=\frac{1}{2}\left[\left(a-q_{i}\right) q_{i}-c q_{i}\right]$. By the first order condition $\frac{\partial u_{i}}{\partial q_{i}}=\frac{1}{2}\left(a-2 q_{i}-c\right)=0, q_{1}^{*}=q_{2}^{*}=\frac{a-c}{2}$.

Therefore, in same type of products with fixed costs, the equilibrium $\left(q_{1}^{*}, q_{2}^{*}\right)=\left(\frac{a-c}{2}, \frac{a-c}{2}\right)$.

A2) With fixed costs. If the costs, which contain the costs of production and the fixed costs, of two enterprises are $c_{1}$ and $c_{2}$, respectively, then the profit function of enterprise $i$ is

$$
u_{i}\left(q_{i}, q_{j}\right)= \begin{cases}\left(a-q_{i}\right) q_{i}-c_{i} q_{i} & q_{i}<q_{j} \\ \frac{1}{4}\left[2\left(a-q_{i}\right) q_{i}-c_{i} q_{i}-c_{j} q_{i}\right] & q_{i}=q_{j}, \\ 0 & q_{i}>q_{j}\end{cases}
$$

where $\{i, j\}=\{1,2\}$. Similar to A1), we have $q_{1}^{*}=q_{2}^{*}$. Thus, the profit of enterprise $i$ is

$$
u_{i}\left(q_{i}, q_{j}\right)=\frac{1}{4}\left[2\left(a-q_{i}\right) q_{i}-c_{i} q_{i}-c_{j} q_{i}\right]
$$

By the first order condition

$$
\frac{\partial u_{i}}{\partial q_{i}}=\frac{1}{4}\left(2 a-4 q_{i}-c_{i}-c_{j}\right)=0
$$

We have $q_{1}^{*}=q_{2}^{*}=\frac{2 a-c_{1}-c_{2}}{4}$. Therefore, in this case, the equilibrium $\left(q_{1}^{*}, q_{2}^{*}\right)=\left(\frac{2 a-c_{1}-c_{2}}{4}, \frac{2 a-c_{1}-c_{2}}{4}\right)$. We can see that, if $c_{1}=c_{2}=c$, the equilibrium is equal to it in A1).

\subsection{Different types of products with same alternative coefficients}

In the pursuing differentiated market, if two enterprises produce different types of products, the market price of the products produced by enterprise $i$ is $p_{i}\left(q_{i}, q_{j}\right)=a-q_{i}+b q_{j}$, where $b>0$ indicates the alternative coefficient between the products produced by enterprise $i$ and enterprise $j$.

B1) Without fixed costs. If the cost of production of both enterprises is $c$, the profit function of enterprise $i$ is $u_{i}\left(q_{i}, q_{j}\right)=\left(a-q_{i}+b q_{j}\right) q_{i}-c q_{i}$, where $\{i, j\}=\{1,2\}$. By the first order condition

$$
\left\{\begin{array}{l}
\frac{\partial u_{1}}{\partial q_{1}}=a-2 q_{1}+b q_{2}-c=0 \\
\frac{\partial u_{2}}{\partial q_{2}}=a-2 q_{2}+b q_{1}-c=0
\end{array},\right.
$$


we have $q_{1}^{*}=q_{2}^{*}=\frac{a-c}{2-b}$. So, in this case, the equilibrium

$$
\left(q_{1}^{*}, q_{2}^{*}\right)=\left(\frac{a-c}{2-b}, \frac{a-c}{2-b}\right)
$$

It is easy to seen that the equilibrium $\left(q_{1}^{*}, q_{2}^{*}\right)=\left(\frac{a-c}{2}, \frac{a-c}{2}\right)$, if the alternative coefficient between the products produced by enterprise 1 and enterprise 2 is 0 , i.e. $b=0$. The result is same to that in case A1).

B2) With fixed costs. If the costs of two enterprises are $c_{1}$ and $c_{2}$, respectively, then the profit function of enterprise $i$ is $u_{i}\left(q_{i}, q_{j}\right)=\left(a-q_{i}+b q_{j}\right) q_{i}-c_{i} q_{i}$, where $\{i, j\}=\{1,2\}$. By the first order condition

$$
\left\{\begin{array}{l}
\frac{\partial u_{1}}{\partial q_{1}}=a-2 q_{1}+b q_{2}-c_{1}=0 \\
\frac{\partial u_{2}}{\partial q_{2}}=a-2 q_{2}+b q_{1}-c_{2}=0
\end{array},\right.
$$

we have $\left\{\begin{array}{l}q_{1}^{*}=\frac{(2+b) a-2 c_{1}-b c_{2}}{4-b^{2}} \\ q_{2}^{*}=\frac{(2+b) a-2 c_{2}-b c_{1}}{4-b^{2}}\end{array}\right.$.

So, in this case, the equilibrium

$$
\left(q_{1}^{*}, q_{2}^{*}\right)=\left(\frac{(2+b) a-2 c_{1}-b c_{2}}{4-b^{2}}, \frac{(2+b) a-2 c_{2}-b c_{1}}{4-b^{2}}\right) .
$$

It can be seen that, if $c_{1}=c_{2}=c$, the equilibrium in this case is same to that in case B1).

\subsection{Different types of products with different alternative coefficients}

In the pursuing differentiated market, if two enterprises produce different types of products with different alternative coefficients, the market price of different types of products is $p_{i}\left(q_{i}, q_{j}\right)=a-q_{i}+b_{i} q_{j}$, where $\{i, j\}=\{1,2\}, b_{i}>0$ indicates the alternative coefficient between the products produced by enterprise $i$ and enterprise $j$.

C1) Without fixed costs. If the cost of production of both enterprises is $c$, the profit function of enterprise $i$ is

$$
u_{i}\left(q_{i}, q_{j}\right)=\left(a-q_{i}+b_{i} q_{j}\right) q_{i}-c q_{i} .
$$

By the first order condition

$$
\left\{\begin{array}{l}
\frac{\partial u_{1}}{\partial q_{1}}=a-2 q_{1}+b_{1} q_{2}-c=0 \\
\frac{\partial u_{2}}{\partial q_{2}}=a-2 q_{2}+b_{2} q_{1}-c=0
\end{array},\right.
$$


we have $\left\{\begin{array}{l}q_{1}^{*}=\frac{\left(2+b_{1}\right)(a-c)}{4-b_{1} b_{2}} \\ q_{2}^{*}=\frac{\left(2+b_{2}\right)(a-c)}{4-b_{1} b_{2}}\end{array}\right.$.

Hence, in this case, the equilibrium

$$
\left(q_{1}^{*}, q_{2}^{*}\right)=\left(\frac{\left(2+b_{1}\right)(a-c)}{4-b_{1} b_{2}}, \frac{\left(2+b_{2}\right)(a-c)}{4-b_{1} b_{2}}\right) .
$$

It is clear that, if $b_{1}=b_{2}=b$, the equilibrium in this case is same to that in case B1).

C2) With fixed costs. If the costs of two enterprises are $c_{1}$ and $c_{2}$, respectively, then the profit function of enterprise $i$ is $u_{i}\left(q_{i}, q_{j}\right)=\left(a-q_{i}+b_{i} q_{j}\right) q_{i}-c_{i} q_{i}$.

By the first order condition

$$
\left\{\begin{array}{l}
\frac{\partial u_{1}}{\partial q_{1}}=a-2 q_{1}+b_{1} q_{2}-c_{1}=0 \\
\frac{\partial u_{2}}{\partial q_{2}}=a-2 q_{2}+b_{2} q_{1}-c_{2}=0
\end{array},\right.
$$

we have $\left\{\begin{array}{l}q_{1}^{*}=\frac{\left(2+b_{1}\right) a-2 c_{1}-b_{1} c_{2}}{4-b_{1} b_{2}} \\ q_{2}^{*}=\frac{\left(2+b_{2}\right) a-2 c_{2}-b_{2} c_{1}}{4-b_{1} b_{2}}\end{array}\right.$.

Hence, in this case, the equilibrium

$$
\left(q_{1}^{*}, q_{2}^{*}\right)=\left(\frac{\left(2+b_{1}\right) a-2 c_{1}-b_{1} c_{2}}{4-b_{1} b_{2}}, \frac{\left(2+b_{2}\right) a-2 c_{2}-b_{2} c_{1}}{4-b_{1} b_{2}}\right)
$$

It can be seen that, if $c_{1}=c_{2}=c$, the result is same to that in case C1); if $b_{1}=b_{2}=b$, the result is same to that in case B2); if $b_{1}=b_{2}=b$ and $c_{1}=c_{2}=c$, the result is same to that in case $\mathrm{B} 1$ ); if $b_{1}=b_{2}=0$, the result is same to that in case A2); if $b_{1}=b_{2}=0$ and $c_{1}=c_{2}=c$, the result is same to that in case $\mathrm{A} 1)$.

\section{Characters of equilibrium in matrix game}

In general form matrix game, for player 1 and player 2, the strategies of player 1 are $U$ and $D$, and the strategies of player 2 are $L$ and $R$. Their profits are as follows:

(1) if player 1 chooses strategy $U$ and player 2 chooses strategy $L$, their profits are $a$ and $b$, respectively;

(2) if player 1 chooses strategy $U$ and player 2 chooses strategy $R$, their profits are $c$ and $d$, respectively;

(3) if player 1 chooses strategy $D$ and player 2 chooses strategy $L$, their profits are $e$ and $f$, respectively;

(4) if player 1 chooses strategy $D$ and player 2 chooses strategy $R$, their profits are $g$ and $h$, respectively. 
It can be shown as the following matrix:

\begin{tabular}{c|c|c|} 
& $\boldsymbol{L}$ & $\boldsymbol{R}$ \\
\hline $\boldsymbol{U}$ & $a, b$ & $c, d$ \\
\hline $\boldsymbol{D}$ & $e, f$ & $g, h$ \\
\hline
\end{tabular}

Where the strategies of player 1 and player 2 are in the first column and the first row respectively, and in the range of profits, the former is player 1's and the latter is player's, respectively.

Given the probabilities of player 1 choosing strategy $U$ and player 2 choosing strategy $L$ be $x$ and $y$, respectively. Thus, the probability of player 1 choosing strategy $D$ is $1-x$, and the probability of player 2 choosing strategy $R$ is $1-y$.

It can be seen that, for player 1 , the expectation of profit of choosing strategy $U$ is $a y+c(1-y)$, and the expectation of profit of choosing strategy $D$ is ey $g(1-y)$. So, there is no difference to choose strategy $U$ and strategy $D$ for player 1 , if and only if $a y+c(1-y)=e y+g(1-y)$. Let $r_{1}(y)$ be the response function of player 1 to the probability $y$ of player 2 . Then

$$
r_{1}(y)=\left\{\begin{array}{cl}
U, & a y+c(1-y)>e y+g(1-y) \\
D, & a y+c(1-y)<e y+g(1-y) . \\
\{U, D\}, & \text { ay }+c(1-y)=e y+g(1-y)
\end{array}\right.
$$

Similarly, for player 2, the expectation of profit of choosing strategy $L$ is $b x+f(1-x)$, and the expectation of profit of choosing strategy $R$ is $d x+h(1-x)$. So, there is no difference to choose strategy $L$ and strategy $R$ for player 2, if and only if $b x+f(1-x)=d x+h(1-x)$. Let $r_{2}(x)$ be the response function of player 2 to the probability $x$ of player 1 . Then

$$
r_{2}(x)=\left\{\begin{array}{cl}
L, & b x+f(1-x)>c x+h(1-x) \\
R, & b x+f(1-x)<c x+h(1-x) . \\
\{L, R\}, & b x+f(1-x)=c x+h(1-x)
\end{array}\right.
$$

Since equilibrium is the optimal response of each player to the others' strategy, the equilibrium of pure strategy, in general form matrix game, has the following characters:

(1) $(U, L)$ is an equilibrium if and only if $a \geq e$ and $b \geq d$;

(2) $(U, R)$ is an equilibrium if and only if $c \geq g$ and $d \geq b$;

(3) $(D, L)$ is an equilibrium if and only if $e \geq a$ and $f \geq h$;

(4) $(D, R)$ is an equilibrium if and only if $g \geq c$ and $h \geq f$.

\section{Acknowledgment}

This paper supported by basic research fund of Xi'an university of architecture and technology (Grant No. JC1709).

\section{References}

[1] Robert Gibbons. A Primer in Game Theory, Information and Control, Prentice Hall, 1994.

[2] Drew Fudenberg and Jean Tirole. Game Theory, MIT Press, 1991.

[3] Weiying Zhang. Game and Society, Peking University press, 2013.

[4] Harold W. K.. Classics in Game theory, Princeton. NJ: Princeton University Pres, 1997.

[5] Myerson, R.. Game Theory: Analysis of Conflict, Cambridge and London: Harvard University Press, 1991.

[6] Osborne, M. and A. Rubinstein. A Course in Game Theory, Cambridge and London: The MIT Press, 1994.

[7] Shanshan Yang. A Study on Bidding Strategy of Power Generation Enterprises in China based 
on Game Theory, Journal of Harbin University of Science and Technology, vol. 22(3), 14-18, 2014. [8] Fulin Li. The Game Between Popular Culture and Elite Culture from the Field Perspective -- a Reflection on Contemporary Chinese Aesthetic Culture, Journal of Shandong Normal University, vol. 17(2), 106-115, 2014. 